\title{
Análisis del perfil socioeconómico y ambiental de la comuna las huacas, archipiélago de jambelí
}

\section{Analysis of the socio-economic and environmental profile of the las huacas community, archipiélago de jambelí}

DOI: $10.46932 / \mathrm{sfjdv2n4-051}$

Received in: March 1st, 2021

Accepted in: May 30th, 2021

\author{
Doménica Belén Sánchez Sarango \\ Estudiante carrera de economía agropecuaria \\ Universidad Técnica de Machala. Km 5 1/2 vía Pasaje \\ Machala-Ecuador \\ E-mail: dsanchez4@utmachala.edu.ec \\ Freddy Patricio Quizhpe Cordero \\ Ingeniero Acuacultor \\ Universidad Técnica de Machala. Km 5 1/2 vía Pasaje \\ Machala-Ecuador \\ E-mail: pquizhpe@utmachala.edu.e \\ Jessica Maribel Quezada Campoverde \\ Ingeniera agrónoma \\ Universidad Técnica de Machala. Km 5 1/2 vía Pasaje \\ Machala-Ecuador \\ E-mail: jquezada@utmachala.edu.ec \\ Víctor Javier Garzón Montealegre \\ Economista \\ Universidad Técnica de Machala. Km 5 1/2 vía Pasaje \\ Machala-Ecuador \\ E-mail:vgarzon@utmachala.edu.ec
}

\section{RESUMEN}

El presente artículo presenta un panorama general sobre el perfil socioeconómico y ambiental de la captura artesanal de conchas (Anadara tuberculosa) en la comuna Las Huacas ubicada en el archipiélago de Jambelí de la provincia de El Oro de Ecuador. El objetivo de la investigación es analizar el perfil socioeconómico y ambiental de la comuna en función de los indicadores de dimensión social, económica y ambiental. Esta investigación obedeció a un diseño no experimental, de campo y transversal, la cual se realizó mediante una ficha técnica de cuestionarios aplicados a los asociados. la población fue dominada por el género masculino (119) sobre el femenino (19); de forma relativa representa un (14\%) la participación de la mujer entre los asociados. Se aplicó la prueba de Chi-cuadrado para determinar significancia $(\alpha<0.05)$ en los indicadores razón de emprender/necesidad u oportunidad $y$ Escolaridad/ingresos promedio. Se concluye que existe una relación de dependencia entre las variables necesidad y oportunidad de emprender, mientras que no existe asociación estadística entre las variables categóricas nivel de instrucción formal e ingresos económicos. Un dato relevante se pudo evidenciar en el indicador ambiental que el 67,15\% de los asociados establecieron que siempre aplican cuidados 
ambientales en la actividad conchera, y el 53,63\% revelaron que siempre realizan actividades de mejora para sanear el medio ambiente.

Palabras clave: Producción, Actividad conchera, concheros.

\begin{abstract}
This article presents an overview of the socioeconomic and environmental profile of the artisanal capture of conch (Anadara tuberculosa) in the Las Huacas commune located in the Jambelí archipelago of the El Oro province of Ecuador. The objective of the research is to analyze the socioeconomic and environmental profile of the commune based on the indicators of social, economic and environmental dimension. This research obeyed a non-experimental, field and cross-sectional design, which was carried out using a technical sheet of questionnaires applied to the associates. the population was dominated by the male gender (119) over the female (19); Relatively, it represents (14\%) the participation of women among the associates. The Chi-square test was applied to determine significance $(\mathrm{p}<0.05)$ in the indicators reason for undertaking / need or opportunity and Schooling / average income. It is concluded that there is a dependency relationship between the variables need and opportunity to undertake, while there is no statistical association between the categorical variables level of formal education and income. A relevant data could be evidenced in the environmental indicator that $67.15 \%$ of the associates established that they always apply environmental care in the shellfish activity, and $53.63 \%$ revealed that they always carry out improvement activities to clean up the environment.
\end{abstract}

Keywords: Production, shell activity, shells.

\title{
1 INTRODUCCIÓN
}

Según Aguilar., et al (2020), la economía ecuatoriana se ha caracterizada a lo largo del tiempo por tener un modelo primario exportador y extractivita. El país ha implementado estrategias en los últimos tiempos para fortalecer la comercialización de productos, y a su vez generen un desarrollo sostenible en el territorio (Quiñónez et al., 2020).

En el país las actividades como la agricultura rural, acuícolas, es decir todas que se encuentren en relación con la tierra, el mar y la naturaleza han contribuido en el crecimiento económico. Sin embargo, las actividades como pesca artesanal, captura de moluscos, crustáceos y demás, que ocupan un 25\% de la captura a nivel mundial han contribuido en la economía del país siendo un sustento de trabajo para muchas familias (Banchón et al., 2020).

Para Muñoz (2018), en el país, la producción marítima de crustáceos, moluscos, peces y otros, desde el año 2016 ha generado ingresos de USD 572.95 millones de dólares aportando alrededor del $0,83 \%$ en el PIB total. Una de las actividades que ha cobrado crecimiento es la pesquería o captura artesanal de moluscos bivalvo (Anadara tuberculosa y la concha A. similis) que son comercializados para consumo. La autora sostiene que esta especie de molusco concentra su presencia en la zona intermareal de los manglares ecuatorianos, y según el Instituto Nacional de Pesca (INP) los lugares don se encuentra la mayor existencia de este molusco son Esmeraldas y El Oro. 
La subsecretaría de Recursos Pesqueros del Ecuador reportó que las actividades como pesca artesanal, captura de moluscos, crustáceos, entre otros registró la captura de alrededor de 40.000 toneladas de pesca al año, las cuales a su vez generaron más de USD 40 y USD 100 millones de dólares por concepto de exportaciones, y en el mercado local respectivamente. Así mismo, para la provincia de El Oro, para las familias dedicadas a estas actividades significó un papel preponderante en el sustento económico (Banchón et al., 2020).

La provincia de El Oro es considera netamente agrícola, y según los reportes, la pesca artesanal involucra a los pescadores de las comunas en situaciones sociales, económicas y ambientales. En tal sentido, es importante identificar las características particulares que evidencien las dimensiones económicas, sociales y ambientales de los pescadores. En base a los mencionado, el objetivo de la investigación es analizar el perfil socioeconómico y ambiental de la comuna las Huacas, que se encuentra ubicada en el archipiélago de Jambelí de la Provincia de El Oro.

\section{METODOLOGÍA}

El presente estudio se desarrolló en la provincia de El Oro que está ubicada en el litoral sur del Ecuador, centrando la investigación en el Archipiélago de Jambelí, donde se encuentra la comuna Las Huacas. La vegetación en la isla está compuesta predominantemente por bosques de manglares (Rhizphophora mangle). La comunidad se encuentra conformada por playas y áreas de vegetación matorral. Estas zonas cuentan con un excelente clima, temperaturas aproximadamente de $24^{\circ} \mathrm{C}$ y una precipitación anual de $500 \mathrm{~mm}$.

La investigación realizada se encuentra dentro del paradigma positivista, por lo tanto, con base a este paradigma y la naturaleza de la información recolectada para responder al problema de esta investigación, la misma es de tipo cualitativa y cuantitativa, así mismo, esta investigación busca determinar, evaluar e interpretar las variables relacionadas con el problema (Tabla 1 y 2), además se intenta encontrar las causas de éste. Esta investigación obedece a un diseño no experimental, de campo, transversal.

La investigación toma como muestra objetivo a las personas de la comuna. El cálculo de la población objetivo se basó en los registros del Instituto Nacional de Estadísticas y Censos INEC (2010) y del Plan de Desarrollo y Ordenamiento Territorial de Jambelí del GAD Santa Rosa, 2015); en donde se obtuvo la siguiente información: Total población en Jambelí: 1718 pobladores, sin embargo, se consideró la cantidad de socios dedicados a la actividad de pesca, recolección de conchas y demás de la comuna que dan un total de 213 personas como población objetivo. 

ÍTEMS

Tabla 1. Ficha técnica de los cuestionarios aplicados a asociados $(\mathrm{N}=213)$.

Unidad de Análisis

Técnica

Tamaño de la muestra

Margen de error

\section{VARIABLES}

\section{Comuna Las Huacas}

Encuestas aplicadas a Asociados.

138 (Tamaño de la muestra para una población conocida) $n=$ $\frac{N * Z^{2} * P * Q}{(N)(E)^{2}+Z^{2} * P * Q}$

Error 5\%

Nivel de confianza del $95 \%$

Tabla 2. Características de los indicadores por dimensión social, económica y ambiental, empleado una encuesta a recolectores concheros de la Comuna Las Huacas.

\begin{tabular}{|c|c|c|c|}
\hline $\mathbf{N}^{\circ}$ & \multicolumn{3}{|c|}{ Social } \\
\hline & Pregunta & $\begin{array}{l}\text { Tipo de } \\
\text { indicador }\end{array}$ & Significado \\
\hline 1 & Género & Dicotómica & Femenino/Masculino \\
\hline 2 & Edad & Intervalo & 18-76 años \\
\hline 3 & Nivel de instrucción & Nominal & $\begin{array}{l}\text { Primaria Completa; Primaria sin concluir; } \\
\text { Secundaria completa; Secundaria sin concluir. }\end{array}$ \\
\hline 4 & Seguro Social & Dicotómica & $\mathrm{Si} / \mathrm{No}$ \\
\hline 5 & Razón de emprender & Dicotómica & Necesidad/Oportunidad \\
\hline 6 & Servicio Básicos & Dicotómica & $\mathrm{Si} / \mathrm{No}$ \\
\hline $\mathbf{N}^{\circ}$ & \multicolumn{3}{|c|}{ Económico } \\
\hline & Pregunta & $\begin{array}{l}\text { Tipo de } \\
\text { indicador }\end{array}$ & Significado \\
\hline 1 & $\begin{array}{l}\text { Productividad (Cantidad de } \\
\text { conchas) }\end{array}$ & Intervalo & $\begin{array}{l}\text { Captura; } 1 \text { a 100, } 101 \text { a 200; } 200 \text { a 300; >300 } \\
\text { conchas }\end{array}$ \\
\hline 2 & Financiamiento & Nominal & $\begin{array}{l}\text { Banca Privada, Banca pública, Cooperativas de } \\
\text { Ahorro y créditos. }\end{array}$ \\
\hline 3 & Ingresos Anuales & Intervalo & $\begin{array}{l}0 \text { a } 2.400 ; 2.401 \text { a } 3400 ; 3401 \text { a } 4.400 ; 4401 \text { a } \\
5400 ;>5400 \text { dólares americanos. }\end{array}$ \\
\hline $\mathbf{N}^{\circ}$ & \multicolumn{3}{|r|}{ Ambiental } \\
\hline & Pregunta & $\begin{array}{l}\text { Tipo de } \\
\text { indicador }\end{array}$ & Significado \\
\hline 1 & $\begin{array}{l}\text { Realizan cuidados } \\
\text { ambientales en la actividad } \\
\text { conchera. }\end{array}$ & Escala & $\begin{array}{l}\text { 1: Nunca; } 2 \text {. Pocas veces; } 3 \text {. A veces sí y a } \\
\text { veces no; } 4 \text {. Muchas veces o muy de acuerdo; } 5 \text {. } \\
\text { Siempre }\end{array}$ \\
\hline 2 & $\begin{array}{l}\text { Realiza actividades de } \\
\text { mejora para sanear el } \\
\text { medio ambiente. }\end{array}$ & Escala & $\begin{array}{l}\text { 1: Nunca; } 2 \text {. Pocas veces; } 3 \text {. A veces sí y a } \\
\text { veces no; } 4 \text {. Muchas veces o muy de acuerdo; } 5 \text {. } \\
\text { Siempre }\end{array}$ \\
\hline
\end{tabular}




\subsection{MÉTODO DE ANÁLISIS DE DATOS}

El cuestionario aplicado fue sometido a un control de calidad, para proceder a codificar y transcribir los datos en formato Excel, donde se utilizó métodos estadísticos para verificar los resultados obtenidos a través del cuestionario empleando en el programa estadístico IBM SPSS Statistics v. 24. En base a los resultados obtenidos se elaboraron gráficas y tablas para su respectiva interpretación.

De esta manera, la presente investigación se realizó en dos momentos secuenciales en el tiempo. En un primer momento, se utilizó la prueba del alfa de Cronbach para determinar la confiabilidad del instrumento. En un segundo momento, se aplicó estadística descriptiva que permitió analizar el comportamiento de las variables mediante los cálculos de porcentajes de participación, distribución de frecuencias, tablas y gráficos.

\section{RESULTADOS}

\subsection{ANÁLISIS DE FIABILIDAD}

Para determinar la confiabilidad del instrumento se realizó una prueba piloto, en donde se encuestó a 138 personas, lo cual, mediante la prueba del Alfa de Cronbach arrojó lo siguientes datos:

Tabla 3. Resumen de las estadísticas de procesamiento y fiabilidad de los casos

\begin{tabular}{|c|c|c|c|c|c|}
\hline \multirow{4}{*}{ Casos } & & $\mathbf{N}$ & $\%$ & \multirow{3}{*}{$\begin{array}{c}\text { Alfa de } \\
\text { Cronbach } \\
0,83\end{array}$} & \multirow{3}{*}{$\begin{array}{c}\mathbf{N}^{\circ} \text { de } \\
\text { elementos } \\
11\end{array}$} \\
\hline & Valido & 138 & 100 & & \\
\hline & Excluido & 0 & 0 & & \\
\hline & Total & 138 & 100 & & \\
\hline
\end{tabular}

Según el estadístico de fiabilidad (Alfa de Cronbach) el instrumento conformado por 11 preguntas con una prueba piloto de 138 encuestados se obtuvo el valor de Alfa de Cronbach de 0.83 . Es decir, esto significa que, el instrumento tiene una confiabilidad muy alta, y por ello servirá para poder recolectar los datos posteriormente.

\subsection{ANÁLISIS ESTADÍSTICO}

Luego de realizar la agrupación de las variables de estudio, se obtuvo las frecuencias y porcentajes respecto a las respuestas elegidas por los 138 asociados de la Comuna Las Huacas. También se elaboró una prueba de hipótesis de Chi cuadro, entre variables nominales y categorías de las diferentes dimensiones, con un nivel de significancia $\alpha<0.05$.

Las hipótesis fueron planteadas en función de: 
Hipótesis 1: La necesidad u oportunidad inciden en la razón de emprender de los asociados de la Comuna Las Huacas.

$\mathrm{H} 0=\mu 1=\mu 2 ; \mathrm{H} 1=\mu 1 \neq \mu 2 \quad$ (Ecuación 2) Siendo: $\mu 1=$ razón de emprender $\mathrm{y} \mu 2=$ necesidad $\mathrm{u}$ oportunidad.

Hipótesis 2: El nivel de instrucción incide el ingreso económico de los asociados de la Comuna Las Huacas.

$\mathrm{H} 0=\mu 1=\mu 2 ; \mathrm{H} 1=\mu 1 \neq \mu 2$ (Ecuación 1 ) siendo: $\mu 1=$ Escolaridad y $\mu 2=$ Ingresos promedios.

\section{RESULTADOS Y DISCUSIÓN}

De acuerdo a la metodología explicada a fin de cumplir con el objetivo de esta investigación, se realizó una encuesta, tomando en cuenta algunas preguntas que son parte de los indicadores que se detallan a continuación:

\subsection{INDICADORES SOCIALES}

En el análisis de los resultados, la relación género y edad se puede apreciar en la Figura 1. En general, la población fue dominada por el género masculino (119) sobre el femenino (19); de forma relativa representa un (14\%) de la participación de la mujer entre los asociados. En el rango de edad de los recolectores masculino se evidencian una gran participación existen de 27 hasta 58 años que se dedican a la captura de los moluscos, mientras que el género femenino se observan intervalos de 27 hasta 42 años. Sin embargo, según (Prado, 2018) la mujer está afianzándose en el área agropecuaria, y, por lo tanto, es necesario que reciba capacitaciones en marketing, comercialización y almacenamiento en molusco. 
Figura 1. Participación de género y edad de los recolectores.

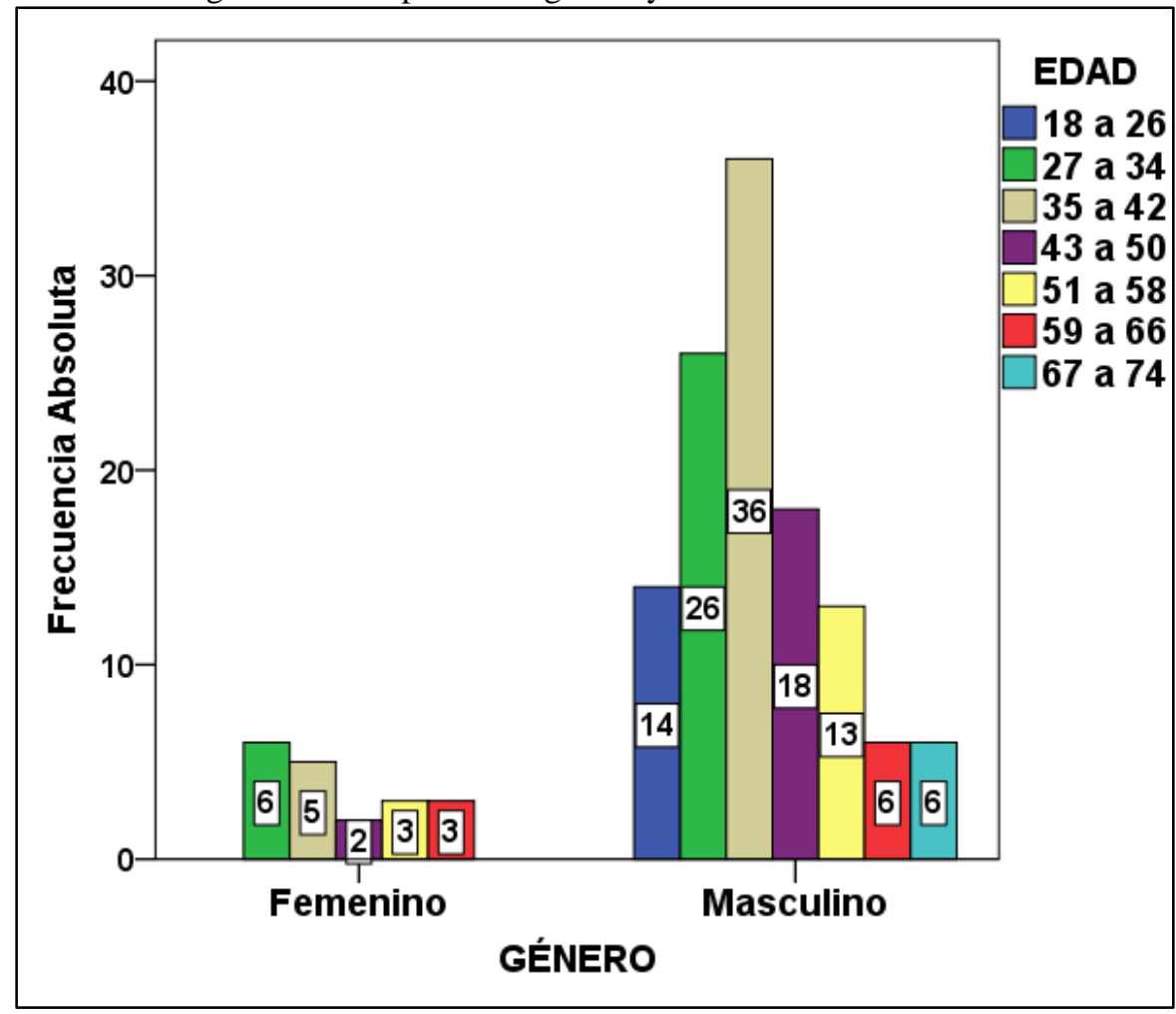

Se indica el nivel de instrucción formal por género, en la Figura 2, de acuerdo a la encuesta aplicada, la mayor frecuencia fue el género masculino presenta más de la mitad de personas que han culminado sus estudios primarios, así mismo 24 personas contestaron no haber culminado sus estudios primarios, mientras que secundaria sin concluir y completa tiene resultados similares, y además tan solo 4 personas mencionaron ser analfabetas.

En cambio, el género femenino fue el de menor frecuencia porque tan solo 11 personas indicaron haber culminados sus estudios primarios y también 4 personas respondieron no haber culminado sus estudios primarios. Es importante señalar, que la mayoría personas que fueron encuestadas indicaron que han finalizado sus estudios primarios, según Prado et al., (2018) establece que con el trascurso del tiempo la Comuna Las Huacas no tendría una mejora en el desarrollo debido a su baja instrucción formal. 
Figura 2. Nivel de instrucción formal por género de los recolectores.

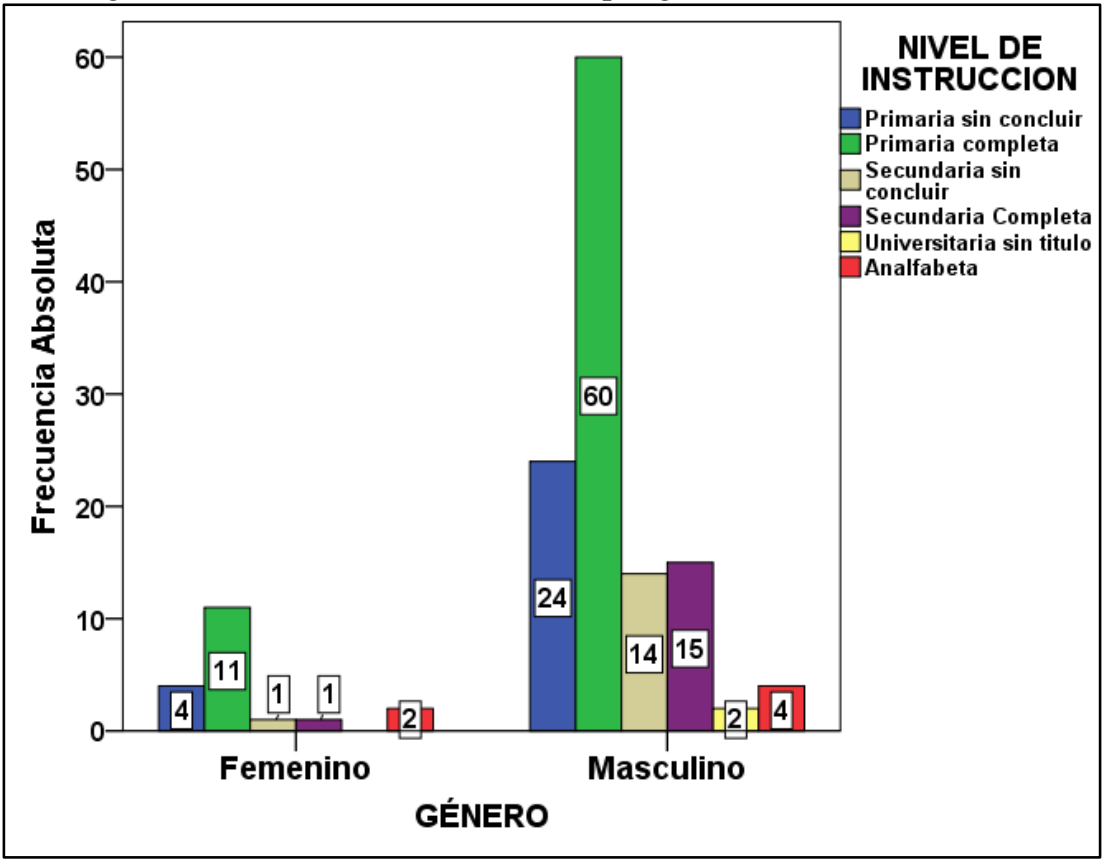

Al visualizar la Tabla 4, podemos encontrar las variables sociales, los comuneros establecen que no cuentan con alcantarillado, telefonía fija, internet fijo y tampoco con un seguro social. Por esta razón, es necesario contar con un servicio de salud o algún Subcentro de salud en la comunidad. Según Ramírez et al., (2020) disponer de una atención primaria de salud y medicina integral permite garantizar de mejor manera la calidad de vida de la población.

Energía eléctrica y agua potable son dos servicios básicos que gran parte de los comuneros tienen a disposición. Además, más de la mitad hacen uso del servicio de telefonía móvil, internet móvil, Tv y Radio.

Tabla 4. Frecuencia de servicios básicos.

\section{Afirmación (\%)}

\begin{tabular}{|c|c|c|c|c|c|c|c|c|c|}
\hline $\begin{array}{c}\text { Variables } \\
\text { sociales }\end{array}$ & $\mathbf{S S}^{1}$ & $\mathbf{E E}^{2}$ & $\mathbf{A} \mathbf{P}^{\mathbf{3}}$ & $\mathbf{A} \mathbf{L}^{4}$ & $\mathbf{T F}^{5}$ & $\mathbf{T M}^{6}$ & $\mathbf{I M}^{7}$ & IF $^{8}$ & $\begin{array}{c}\text { TV Y } \\
\text { RADIO }^{9}\end{array}$ \\
\hline Si & 95 & 100 & 100 & 0 & 0 & 95 & 95 & 0 & 88 \\
\hline No & 5 & 0 & 0 & 100 & 100 & 5 & 5 & 100 & 12 \\
\hline
\end{tabular}

\footnotetext{
${ }^{1}$ Seguro Social $;{ }^{2}$ Energía Eléctrica; ${ }^{3}$ Agua Potable; ${ }^{4}$ Alcantarillado; ${ }^{5}$ Telefonía Fija; ${ }^{6}$ Telefonía Móvil; ${ }^{7}$ Internet Móvil; ${ }^{8}$ Internet Fijo; ${ }^{9} \mathrm{Tv}$ y Radio.

Elaboración: Por autores.
} 


\subsection{INDICADORES ECONÓMICOS}

El indicador económico ingresos y fuentes de financiamiento se presenta en la Figura 3. Dentro del intervalo de ingresos económicos mayor a \$5.400 anuales, se registró a 102 asociados, es decir, la mayoría de los comuneros al final del año registran esos valores. La principal fuente de financiamiento provino del sector público $(53,62 \%)$ de los asociados, seguidos de las cooperativas de ahorro y crédito $(5,07 \%)$ de asociados que solicitaron préstamos. En el caso de las otras fuentes de financiamiento $(28,26 \%)$ y la banca privada $(13,04 \%)$ solo solicitaron financiamiento a estas fuentes.

En la Figura 3, el $(73,91 \%)$ de los recolectores encuestados respondieron que sus ingresos anuales son mayores a $\$ 5.400$, el $(13,77 \%)$ generan ingresos entre $\$ 4.401$ a $\$ 5.400$, y el $(8,70 \%)$ tienen valores entre $\$ 3.401$, y finalmente un $(3,62 \%)$ son los de menores ingresos generado y estos oscilan entre $\$ 2.401$ a \$3.400 dólares americanos. El nivel de ingresos de los asociados en la investigación, está en relación con el tamaño metros lineales recorridos. Carpio et al., (2020) expresan que la conducta económica de los recolectores de crustáceo y molusco está inducida por la adquisición de maquinarias (motores fuera de borda) y otros muebles e inmuebles (botes, pangas, lanchas), como componentes de su principal actividad productiva.

Figura 3. Ingresos (\$) promedios ha/anual y Fuentes de financiamiento.

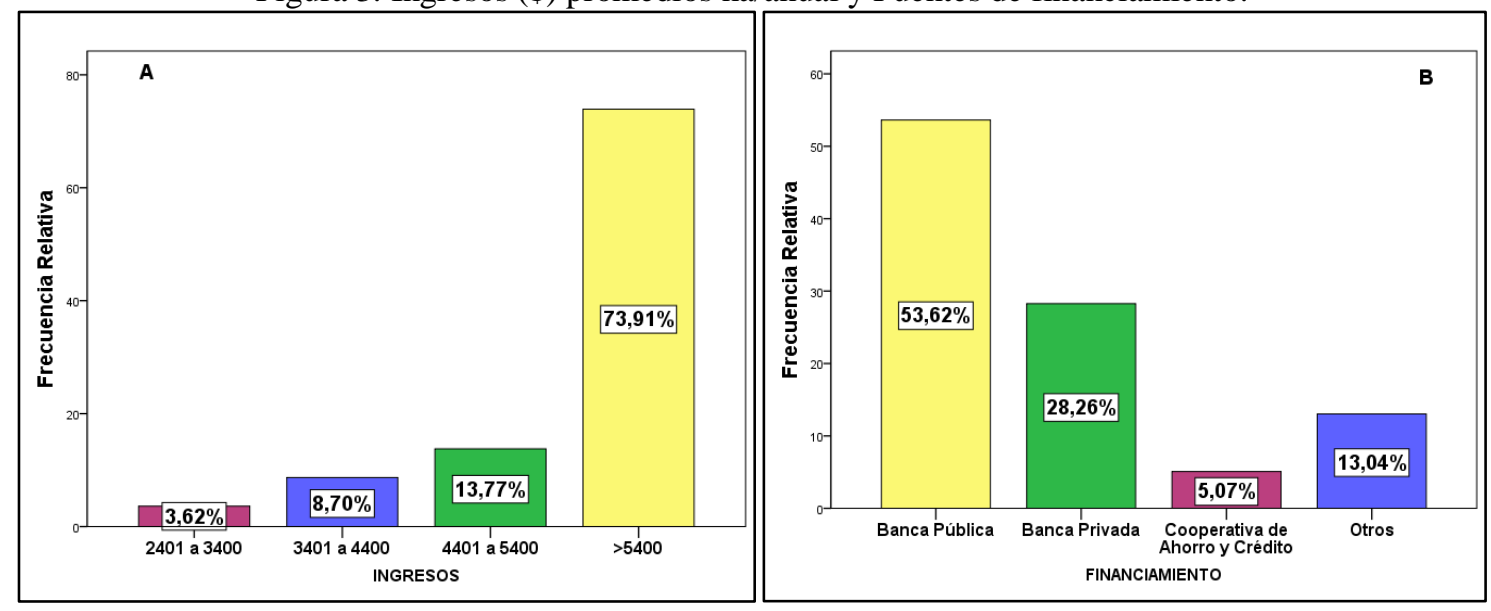

\subsection{INDICADORES AMBIENTALES}

De acuerdo a los resultados obtenidos, se puede visualizar mediante la Figura 4, una muestra de 138 socios que están vinculados en el área de producción del molusco bivalvo "Concha Prieta” contestaron a la pregunta planteada "Realizan cuidados ambientales en la actividad conchera.", de la siguiente forma: Se evidencia que 93 personas representan el $(67,15 \%)$ establecen que siempre se aplica cuidados ambientales en la actividad cochera. Un estudio realizado por Prado-Carpio et al., (2020) afirma que el recurso conchero es una actividad social y económica que se ha desarrollado históricamente en armonía y preservando el ecosistema del manglar, sin embargo, el incremento de la granja acuícola ha incidido en 
la eliminación en grandes superficies del ecosistema de manglar y el deterioro de sus condiciones naturales.

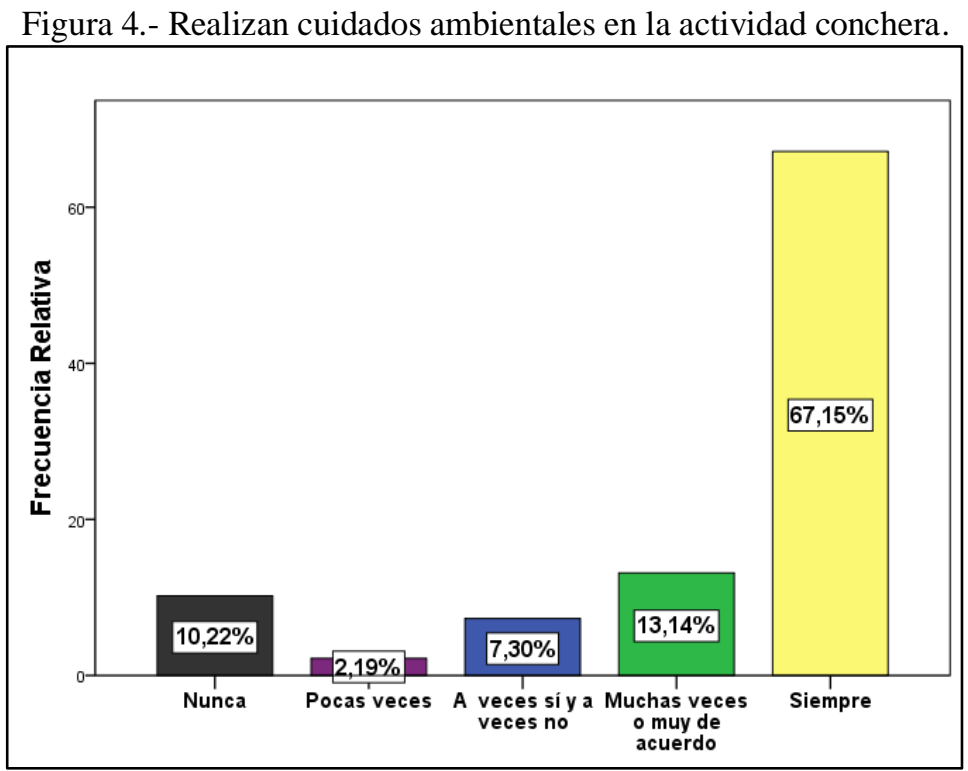

Los resultados de la pregunta "Realiza actividades de mejora para sanear el medio ambiente." se los puede apreciar en la Figura 5, las 138 personas que son parte de la producción del recurso conchero contestaron la pregunta planteada de la siguiente forma: Se evidencia que 88 personas $(53,62 \%)$ tiene la mayor representación, los mismo establecieron que siempre se realiza actividades de mejora para sanear el medio ambiente. Según Mera, (2021) la preservación y manejo del manglar lo representa el incremento en la captura del recurso molusco y crustáceos, esto permite su aprovechamiento económico y social con un mínimo impacto ambiental. 
Figura 5.- Realiza actividades de mejora para sanear el medio ambiente.

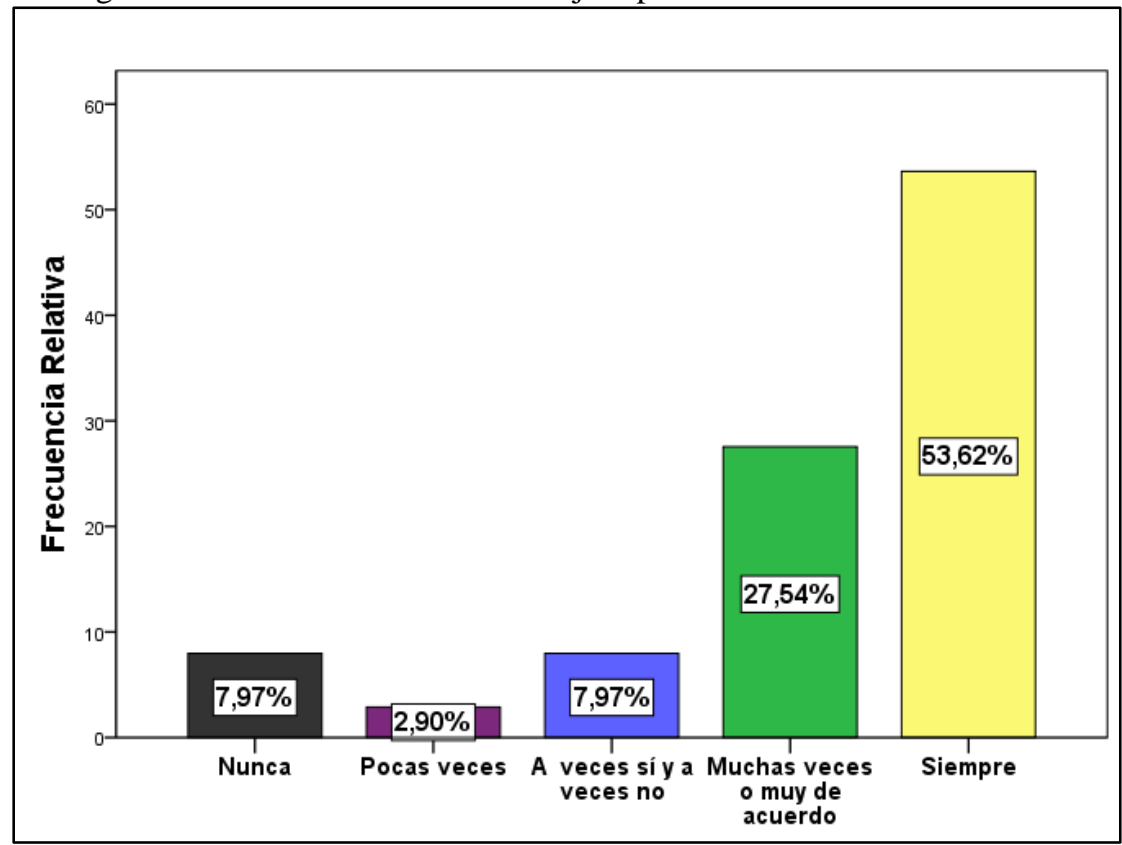

\section{Hipótesis 1}

Tabla 5. Tabla de contingencia de género y razón de emprender.

\begin{tabular}{llcc}
\hline & & \multicolumn{2}{c}{ Género } \\
\cline { 3 - 4 } Razón de emprender & Necesidad & Masculino & Femenino \\
\cline { 2 - 4 } & Oportunidad & $50,20 \%$ & $20.00 \%$ \\
\hline Total & & $100,00 \%$ & $80,00 \%$ \\
\hline
\end{tabular}

Los resultados del presente trabajo en Tabla 5, revelan que, del total de encuestados, se logró constatar que la oportunidad de emprender del género femenino es del (80\%), coincidiendo con lo planteado por Prado-Carpio et al., (2018). Así mismo, el género masculino-oportunidad de emprender es de $(58,80 \%)$, seguido por la necesidad de emprender que es de $(40,20 \%)$. Esto indica un panorama favorable para la implementación de futuros agro negocios en la Comuna Las Huacas.

Tabla 6. Prueba Chi-Cuadrado

\begin{tabular}{lccc}
\multicolumn{1}{c}{ Variables } & Valor & gl & \multicolumn{2}{c}{ Significación asintótica (bilateral) } \\
\hline Chi-cuadrado de Pearson & $42,123^{\text {a }}$ & 18 & 0,001 \\
\hline N de casos válidos & 138 & & \\
\hline
\end{tabular}

Se ha denotado claramente en la Tabla 5 que se obtuvo p-valor (Sig. asintótica bilateral) 0,001 menor al alfa predefinido 0,05, según el cual se rechaza la hipótesis nula. Por esta razón, denotamos que 
existen indicios de una relación de dependencia entre las variables necesidad y oportunidad de emprender, de esta manera, se puede concluir con un nivel de confianza de $95 \%$ que ambas variables son dependientes.

\section{Hipótesis 2}

Tabla 7. Tabla de contingencia ingresos promedios de la actividad-nivel de instrucción formal.

\begin{tabular}{|c|c|c|c|c|c|}
\hline \multirow[t]{2}{*}{ Nivel de instrucción } & \multicolumn{4}{|c|}{ Ingresos } & \multirow[t]{2}{*}{ Total $(\%)$} \\
\hline & 2401 a 3400 & 3401 a 4400 & 4401 a 5400 & $>5400$ & \\
\hline Primaria sin concluir & 0,00 & 0,72 & 2,90 & 16,67 & 20,29 \\
\hline Primaria completa & 2,17 & 5,80 & 7,97 & 35,51 & 51,45 \\
\hline Secundaria sin concluir & 1,45 & 0,72 & 0,00 & 8,70 & 10,87 \\
\hline Secundaria Completa & 0,00 & 0,72 & 1,45 & 9,42 & 11,59 \\
\hline Universitaria sin titulo & 0,00 & 0,00 & 0,00 & 1,45 & 1,45 \\
\hline Analfabeta & 0,00 & 0,72 & 1,45 & 2,17 & 4,35 \\
\hline
\end{tabular}

Se evidencia según los resultados alcanzados, el $(51,45 \%)$ de los recolectores han culminado sus estudios primarios, y a su vez se puede observar que este mismo grupo de personas tiene mejores ingresos que las demás personas que no han culminado sus estudios primarios, secundarios y universitarios. Lizano et al., (2021) obtuvo resultados similares, y afirma que existen negocios agropecuarios que muchas de sus empresarios o productores carecen de estudios secundarios o universitarios, sin embargo, muchos de ellos han mejorado su situación social y económica mediante su agronegocio.

Tabla 8. Prueba Chi-Cuadrado

\begin{tabular}{lclc}
\hline \multicolumn{1}{c}{ Variables } & Valor & gl & \multicolumn{2}{c}{ Significación asintótica (bilateral) } \\
\hline Chi-cuadrado de Pearson & $13,551^{\mathrm{a}}$ & 15 & 0,560 \\
\hline N de casos válidos & 138 & &
\end{tabular}

Se estableció que no existe asociación estadística entre las variables categóricas nivel de instrucción formal e ingresos económicos. Se obtuvo significancia $(\mathrm{p}=0,560)$. De esta manera, se puede concluir con un nivel de confianza de $95 \%$ que ambas variables son independientes.

\section{CONCLUSIONES}

El análisis del perfil socioeconómico y ambiental de la Comuna Las Huacas del Archipiélago de Jambelí, que las personas que tienen mayor la captura del recurso conchero tienen rango de edad 27 a 50 años. 
Los resultados alcanzados, se establecen que existe una relación de dependencia entre las variables necesidad y oportunidad de emprender. Mientras que otro análisis se observa que no existe asociación estadística entre las variables categóricas nivel de instrucción formal e ingresos económicos.

Asimismo, se evidencia características comunes encontradas en otras investigaciones como el predominio del género masculino, los comuneros no cuentan con un seguro social, y la falta de los servicios básicos como alcantarillado y telefonía en la dimensión social. 


\section{BIBLIOGRAFÍA}

Aguilar, B., Illicachi, G., \& Abarca, A. (2020). ANÁLISIS DEL PERFIL SOCIO ECONÓMICO Y SUS EFECTOS EN EL DESARROLLO POBLACIONAL DE ALAUSÍ-ECUADOR. UNIVERSIDAD, CIENCIA y TECNOLOGÍA, 63 - 69. doi:10.47460/uct.v24i107.415

Banchón, A., Vite, C., \& Carvajal, H. (2020). SOCIOECONOMIC AND ENVIRONMENTAL PROFILE OF ARTISANAL FISHING IN THE HUAYLA RIVER COM-MUNITY. Remca, 3, 199 - 205. Obtenido de https://remca.umet.edu.ec/index.php/REMCA/article/view/288/318

Carpio, E. P., Soto, M. E. M., Díaz, A. M., Armijos, C. C., Minuche, P. R., Reyes, J. C., \& Monroy, C. R. (2020). Importancia de la producción de la concha prieta (Anadara tuberculosa) en las costas ecuatorianas. Revista ESPAMCIENCIA ISSN $1390-8103, \quad$ 34-46. http://190.15.136.171:3914/index.php/Revista_ESPAMCIENCIA/article/view/209

Chungata Campoverde, A. E., \& Chungata Jimenez, K. E. (2019). Perfil socioeconómico y ambiental de la asociación agraria bananera fincas de El Oro. http://186.3.32.121/handle/48000/15174

GAD Santa Rosa. (2015). PLAN DE DESARROLLO Y ORDENAMIENTO TERRITORIAL DE JAMBELÍ.

http://app.sni.gob.ec/sni-

link/sni/PORTAL_SNI/data_sigad_plus/sigadplusdiagnostico/0760030760001_DIAGNOSTICO_ACT

UALIZADO_30-10-2015_13-20-06.pdf

INEC. (2010). FASCÍCULO PROVINCIAL EL ORO. https://www.ecuadorencifras.gob.ec/wpcontent/descargas/Manu-lateral/Resultados-provinciales/el_oro.pdf

Lizano, V. R., Vega, M. M., \& Vega, R. M. (2021). Un abordaje multinomial integral para entender la sucesión familiar agrícola en Costa Rica. e-Agronegocios, 7(1), 95-110. https://doi.org/10.18845/ea.v7i1.5390

Mera, G. M. M. (2021). Percepción de los servicios ambientales de provisión en la reserva natural Pacoche. Telos: Revista de Estudios Interdisciplinarios en Ciencias Sociales, 23(2), 267285.https://doi.org/10.36390/telos232.05

Muñóz, C. (2018 de 2018). DIAGNOSTICO SOCIOECONOMICO DEL AGRONEGOCIO CONCHERO (ANADARA TUBERCULOSA) EN LA COMUNA LAS HUACAS DEL ARCHIPIELAGO DE JAMBELI. Repositorio UTMACH. Obtenido de http://repositorio.utmachala.edu.ec/bitstream/48000/13255/1/DE00010_TRABAJODETITULACION.p df

Prado-Carpio, E., Castro-Armijos, C. J., Castillo-Herrera, S., Quezada-Abad, C., Coronel-Reyes, J., \& Arias-Chiriboga, H. (2018). La Equidad De Género En Los Agronegocios: Sector Comercio De Insumos Agropecuarios Y Servicios Veterinarios Del Cantón Machala. European Scientific Journal, ESJ, 14(8). https://doi.org/10.19044/esj.2018.v14n8p\%p 
Prado-Carpio, E., Soto, M. E. M., Rodriguez-Monroy, C., \& Núñez-Guerrero, Y. (2020). Quantification of the Management Model of the Production Chain of the Concha Prieta "Anadara Tuberculosa." EasyChair. http://laccei.org/LACCEI2020-VirtualEdition/full_papers/FP469.pdf

Quiñónez, M., Nazareno, I., Camacho, R., \& Cedeño, M. (2020). Proceso de comercialización y extracción de productos de manglar, San Lorenzo-Ecuador. Revista Venezolana de Gerencia, 91. doi:https://doi.org/10.37960/rvg.v25i91.33172

Ramírez, K. R., Castro, K. V., \& Reyes, J. C. (2020). Pronóstico de profesionales de la salud en la provincia del Oro, Ecuador. Polo Del Conocimiento: Revista Científico-Profesional, 5(1), 542-562. https://dialnet.unirioja.es/servlet/articulo?codigo=7436043 